\title{
Pathogenesis of Thrombotic Microangiopathy: Insights from Animal Models
}

\author{
Anna Richards ${ }^{a}$ David Kavanagh ${ }^{b}$ \\ ${ }^{a}$ MRC Centre for Inflammation Research, Queens Medical Research Institute, Edinburgh, and bepartment of \\ Nephrology, The Institute of Human Genetics, Newcastle University, Newcastle upon Tyne, UK
}

\section{Key Words}

Thrombotic microangiopathy $\cdot$ Haemolytic uraemic syndrome $\cdot$ Thrombotic thrombocytopenic purpura • Vascular endothelial growth factor - Bevacizumab - Mouse models · Complement factor $\mathrm{H} \cdot$ ADAMTS $13 \cdot$ Eculizumab

\begin{abstract}
Animal models are important experimental tools for investigating the molecular mechanisms, environmental and genetic susceptibilities underlying the development of thrombotic microangiopathies. Large mammal, small animal models, knockout, transgenic and conditional knockout mouse models are available to investigate haemolytic uraemic syndrome, thrombotic thrombocytopenic purpura and vascular endothelial growth factor-associated thrombotic microangiopathy. These models have shown that it is possible to model the human conditions. However, differences in human and rodent physiology mean that caution is required when interpreting the findings. These models offer realistic prospects for identifying and testing novel therapeutic strategies in a range of thrombotic microangiopathies prior to human trials.

Copyright $\odot 2009$ S. Karger AG, Basel
\end{abstract}

\section{Thrombotic Microangiopathies}

Thrombotic microangiopathies (TMAs) are a group of conditions characterised by microvascular thrombosis leading to thrombocytopenia, haemolytic anaemia and red cell fragmentation [1]. The clinical manifestation of TMA is determined by the specificity of the process for different endothelial cell beds. This led to the historical differentiation between haemolytic uraemic syndrome (HUS) [2] and thrombotic thrombocytopenic purpura (TTP) [3]. For example, in TTP there is predominantly brain microvascular endothelial cell injury, resulting in neurological disturbance, while in HUS there is primarily glomerular endothelial cell injury resulting in acute renal impairment.

Many different aetiological predisposing factors for the development of TMA have been described. The postdiarrhoeal form of HUS (D+ HUS) is most commonly precipitated by Shiga-toxin (Stx)-producing Escherichia coli. Other precipitating factors for TMA include: Streptococcus pneumoniae infection, pregnancy, drugs (bevacizumab, mitomycin) and malignancy. However, TMAs only manifest in a small proportion of patients exposed to these environmental stimuli, suggesting additional genetic predispositions. Recent advances in molecular biology have begun to identify these factors. TTP has been shown to be predisposed to by a genetic deficiency of

Anna Richards

MRC Centre for Inflammation Research, Queens Medical Research Institute

47 Little France Crescent

Edinburgh EH16 4TJ (UK)

Tel. +44 131242 6654/6562, Fax +44 131242 6578, E-Mail anna.richards@ed.ac.uk 
Table 1. Toxin models of TMAs

\begin{tabular}{|c|c|c|c|}
\hline Model & Trigger & Pathology & Ref. \\
\hline \multicolumn{4}{|l|}{ Shiga toxins } \\
\hline Mouse & oral E. coli O157:H7; Sm & renal cortical tubular necrosis & Wadolkoski et al. (1990) \\
\hline Mouse & IV/IP Stx1, Stx2 & necrosis of tubular cells & Tesh et al. (1993) \\
\hline Mouse & IG E. coli O157:H7 & tubular necrosis & Karpman et al. (1997) \\
\hline Mouse & SQ IP LPS + IV Stx2 & tubular necrosis & Palermo et al. $(1999,2000)$ \\
\hline Mouse & IV Stx2 & widespread mesangial hypercellularity, cresent formation & Dran et al. (2002) \\
\hline Mouse & IV Stx2 + L-NAME & $\begin{array}{l}\text { widespread mesangial hypercellularity, cresent formation, } \\
\text { glomerular thrombosis, tubular necrosis }\end{array}$ & Dran et al. (2002) \\
\hline Mouse & IV Stx1, Stx2 & necrosis of tubular cells & Rutjes et al. (2002) \\
\hline Mouse & SQ IP LPS + Stx2 & mild glomerular endothelial damage & Ikeda et al. (2004) \\
\hline Mouse & IP LPS + Stx 2 & thrombocytopenia, haemolytic anaemia, renal failure, fibrin thrombi & Keepers et al. $(2006,2007)$ \\
\hline Mouse & IP Stx2 & haemolysis, renal failure, glomerular fibrin deposition & Sauter et al. (2008) \\
\hline Mouse & $\begin{array}{l}\text { oral enterohemorrhagic E. coli } \\
\text { germ-free mice }\end{array}$ & $\begin{array}{l}\text { renal tubular necrosis, glomerular capillary RBC sludging and } \\
\text { occasional fibrin thrombi }\end{array}$ & Eaton et al. (2008) \\
\hline Rat & IV Stx2 & acute tubular injury & Zhao et al. (2002) \\
\hline Rat & IP E. coli Stx2 supernatant & tubular + glomerular necrosis, glomerular fibrin thrombi & Zotta et al. (2008) \\
\hline Ferret & oral E. coli O157:H7; Sm & some develop glomerular fibrin thrombi, occasionally thrombocytopenia & Woods et al. (2002) \\
\hline Dutch belted rabbits & oral E. coli $\mathrm{O} 153$ & glomerular fibrin thrombi + tubular necrosis & Garcia et al. (2002) \\
\hline Gnotobiotic piglets & oral E. coli O157:H7 & glomerular TMA, no renal failure, no thrombocytopenia & Gunzer et al. (2002) \\
\hline Greyhounds & oral E. coli O157:H7 & glomerular TMA & Hertzke et al. (1995) \\
\hline \multirow[t]{2}{*}{ Baboon } & IV Stx1, Stx2 & renal TMA & Taylor et al. (1999) \\
\hline & & thrombocytopenia, MAHA, renal failure & Siegler et al. (2003) \\
\hline \multicolumn{4}{|l|}{ Endothelial toxins } \\
\hline Mouse & $\mathrm{IV} / \mathrm{IP}$ ricin \pm LPS & $\begin{array}{l}\text { thrombocytopenia, haemolytic anaemia, renal failure, glomerular } \\
\text { fibrin thrombi }\end{array}$ & Taylor et al. (1999) \\
\hline Mouse & $\begin{array}{l}\text { transplantation of hybridoma } \\
\text { clone (anti-platelet } \mathrm{Ab} \text { ) }\end{array}$ & thrombocytopenia, microthrombi in small vessels of lung & Hashimoto et al. (2000) \\
\hline Mouse & SQ RAP Con A + anti-Con A Ab & glomerular + peritubular microvascular thrombosis + tubular necrosis & Hohenstein et al. (2008) \\
\hline Rat & mitomycin perfusion of kidney & renal TMA & Cattell et al. (1985) \\
\hline Rat & RAP anti-GEC Ab & renal TMA & Nangaku et al. (1997) \\
\hline Rat & IV anti-EC Ab & $\begin{array}{l}\text { thrombocytopenia, tubular injury, occasional glomerular intracapillary } \\
\text { thrombi }\end{array}$ & Ren et al. (2002) \\
\hline \multicolumn{4}{|l|}{ Other } \\
\hline Dog/pig & botrocetin & platelet microthrombi in lungs and spleen & Sanders et al. (1995) \\
\hline Rats & botrocetin & platelet microthrombi in lungs and spleen & Sanders et al. (1988) \\
\hline
\end{tabular}

Sm = Streptomycin pre-treated; IV = intravenous; IP = intraperitoneal; LPS = lipopolysaccharide; SQ = sequential administration; IG = intragastric; $\mathrm{GEC}$ = glomerular endothelial cell; $\mathrm{EC}=$ endothelial cell; RAP = renal artery perfusion; $\mathrm{RBC}=$ red blood cell; TMA = thrombotic microangiopathy.

ADAMTS13 (the rare Upshaw-Schulman syndrome) or more commonly from production of inhibitory antiADAMTS13 antibodies [3]. By contrast, the non-diarrhoeal form of HUS, atypical HUS (aHUS), is predisposed to by mutations in, or antibodies against, complement regulatory proteins [2].

Discovery of these genetic predispositions has allowed generation of specific knockout and transgenic mouse models to add to existing Stx models of TMA. These are now yielding significant advances in the understanding of disease pathogenesis and provide models to screen potential therapeutic agents. The strengths and possible limitations of this experimental approach are reviewed here.

\section{Animal Models of Thrombotic Microangiopathies}

(tables 1, 2)

Initial attempts to generate animal models of thrombotic microangiopathies focused on oral administration of Stx-producing E. coli or parenteral administration of Stx.

\section{Non-Rodent Models of D+HUS}

Greyhound - Shiga Toxin

'Alabama rot' is a naturally occurring model of $\mathrm{D}+$ HUS which was first recognised at a greyhound race track in Alabama. Racing greyhounds are fed raw meat from rendering plants which commonly contains pathogenic 
Table 2. Genetically modified models of TMAs

\begin{tabular}{|c|c|c|c|}
\hline Model & Trigger & Pathology & Ref. \\
\hline \multicolumn{4}{|l|}{ Complement k/o } \\
\hline Norwegian Yorkshire pig & spontaneous & MPGN & Hegasy et al. (2002) \\
\hline Mouse $\mathrm{CFH}^{-/-}$ & spontaneous & MPGN & Pickering et al. (2002) \\
\hline Mouse $\mathrm{CFH}^{+/-}$ & IP Stx2 + LPS & $\begin{array}{l}\text { no TMA, no difference in tubular injury } \\
\text { compared to controls }\end{array}$ & Paixao-Cavalcante et al. (2009) \\
\hline Mouse $\mathrm{CFH}^{-/-} \Delta 16-20$ & spontaneous & aHUS & Pickering et al. (2007) \\
\hline Mouse $\mathrm{C}^{-/-} \mathrm{CFH}^{-/-} \Delta 16-20$ & spontaneous & no aHUS & Goicoechea de Jorge et al. (2008) \\
\hline Mouse $\mathrm{CFI}^{-/-}$ & spontaneous & mesangial C3 deposits with nodular expansion & Rose et al. (2008) \\
\hline \multicolumn{4}{|l|}{ ADAMTS 13 k/o } \\
\hline Mouse ADAMTS $13^{-/-}(129 / \mathrm{Sv})$ & $\mathrm{N} / \mathrm{A}$ & none & Banno et al. (2006) \\
\hline Mouse ADAMTS $13^{-/-}(\mathrm{C} 57 \mathrm{BL} / 6 ; 129 \times 1 / \mathrm{Sv})$ & $\mathrm{N} / \mathrm{A}$ & none & Motto et al. (2005) \\
\hline Mouse ADAMTS $13^{-1-}(\mathrm{CASA} / \mathrm{Rk})$ & spontaneous & TTP like lesions (in some) & Motto et al. (2005) \\
\hline Mouse ADAMTS $13^{-/-}(\mathrm{CASA} / \mathrm{Rk})$ & IV Shiga toxin & TTP like lesions (in most) & Motto et al. (2005) \\
\hline \multicolumn{4}{|l|}{$V E G F k / o$} \\
\hline Mouse VEGFA ${ }^{-/-}$ & spontaneous & major vascular defects & Carmeliet et al. (1996) \\
\hline Mouse podocyte VEGFA ${ }^{-/-}$ & spontaneous & perinatal death, grossly abnormal glomeruli & Eremina et al. (2003) \\
\hline Mouse conditional podocyte VEGFA ${ }^{-/-}$ & spontaneous & TMA & Eremina et al. (2008) \\
\hline \multicolumn{4}{|l|}{ Renin angiotensin $T G$} \\
\hline $\mathrm{R}^{+} / \mathrm{A}^{+}$ & high salt diet/L-NAME & glomerular fibrin thrombi, mesangiolysis & Sethi et al. (2006) \\
\hline
\end{tabular}

E. coli. These dogs develop bloody diarrhoea, skin ulcers and renal failure. The renal pathology is of an extensive glomerular TMA. Preliminary experiments using this model demonstrated that treatment with Lepirudin, a recombinant hirudin anticoagulant that binds thrombin, prevented the lethal effects of Stx2.

\section{Baboon - Shiga Toxin}

Siegler et al. [4] developed a baboon model of Stx-mediated HUS. Animals given IV Stx1 or Stx 2 developed progressive thrombocytopenia, haemolytic anaemia and renal failure. Glomerular thrombotic microangiopathy was found at necropsy. This model demonstrated that Stx2 is a more potent initiator of D+ HUS than Stx1.

\section{Rodent Models of D+ HUS}

Small Animal Models - Shiga Toxin

Thus, canine and baboon models of HUS mimic the human disease; however, they are impractical and costly. Small animal models of Stx HUS have proved a poor homologue of human disease, with most developing tubular damage without glomerular thrombosis (table 1). This is felt to relate to interspecies variability in the expression of the Stx receptor, Gb3, which is absent in mouse glomeruli. However, murine renal tubules do express Gb3, which provides an explanation for the pattern of disease in mice exposed to Stx.
Keepers et al. [5] recently demonstrated that intraperitoneal co-injection of purified Stx2 plus lipopolysaccharide in mice resulted in the classical triad of thrombocytopenia, haemolytic anaemia and renal failure seen in human HUS. However, it has been suggested that this glomerular pathology could follow primary tubular damage [6].

\section{Endothelial Cell Toxin Model of TMA}

Mitomycin C, an alkylating chemotherapy agent, is thought to cause renal thrombotic microangiopathy by a direct toxic effect on endothelium. When mitomycin was perfused into kidneys of rats, a pathological picture resembling HUS resulted, with fibrin deposition and endothelial cell proliferation.

\section{Antibody-Mediated Models of TMA}

Nangaku et al. [7] generated a rat model of TMA that histologically and clinically resembles HUS by selective unilateral renal artery perfusion of antibodies against glomerular endothelial cells. This injury was prevented by using rats that had undergone complement depletion using cobra venom factor or were genetically C6-deficient, suggesting a role for membrane attack complex formation in the pathogenesis. Additional studies using this model also suggested a role for C5b-9-mediated endothelial cell apoptosis and showed protective effects of VEGF.

Nephron Exp Nephrol 2009;113:e97-e103 
A further rat model of TMA with renal disease was developed by Ren et al. [8], using a complement-fixing antibody to endothelial cells. EC injury was prevented by complement depletion and worsened by blockade of Crry. Hashimoto et al. [9] generated a hybridoma clone in lupus-prone mice (MRL/lpr) which, when transplanted into syngeneic non-autoimmune mice, caused microvascular intraluminal platelet aggregation, thrombocytopenia and anaemia. This pathogenic autoantibody (antigp70) specifically precipitated a platelet protein with an approximate relative molecular mass of $40 \mathrm{kDa}$. These studies replicate human studies that have demonstrated the presence in patients' plasma of IgG antibodies reactive with a platelet and/or endothelial cell antigen, especially CD36 in HUS and TTP.

\section{Specific Pathophysiological Models}

\section{Complement Mouse Models of aHUS}

Mutations in the complement regulatory proteins have been shown to predispose to aHUS [2]. This has provided alternative targets for the generation of mouse models of aHUS. Mutations in the complement regulator factor $\mathrm{H}$ have been linked to two different human renal diseases: membranoproliferative glomerulonephritis (MPGN) and aHUS. Although both are associated with complete factor $\mathrm{H}$ deficiency, aHUS is more commonly associated with heterozygous mutations in the C-terminal of $\mathrm{CFH}$ resulting in normal plasma levels of $\mathrm{CFH}$.

To investigate the role of factor $\mathrm{H}$ in disease, Pickering et al. [10] developed a mouse $\mathrm{CFH}$ knockout. This $\mathrm{CFH}^{-/-}$ mouse, which demonstrated uncontrolled turnover of the alternative pathway with very low $\mathrm{C} 3$ levels, developed MPGN, not aHUS. A spontaneously arising $\mathrm{CFH}$ deficient strain of pig has also been shown to develop MPGN, not aHUS.

In order to better mimic the situation seen in aHUS, Pickering et al. [11] generated a transgenic mouse on the background of the $\mathrm{CFH}^{-/-}$mouse which lacked the 5 C-terminal CCPs of $C F H\left(C F H^{-1-} \Delta 16-20\right)$. It is this region, shown to be important for cell-surface binding [12], which contains $80 \%$ of the factor $\mathrm{H}$ mutations reported to cause human aHUS.

This $\mathrm{CFH}^{-l-} \Delta 16-20$ mouse, which had higher plasma C3 levels compared to the $\mathrm{CFH}^{-/-}$mouse, developed aHUS, not MPGN. Thus, this mouse model provides the first in vivo evidence that the $\mathrm{CFH}$ mutations seen in aHUS impair endothelial cell surface recognition, resulting in local complement dysregulation, while controlling the alternative pathway in plasma (reviewed in Pickering et al. [13]). In these mice only the homozygous transgenic mice developed HUS, whereas in humans a heterozygous mutation is sufficient to predispose to aHUS. This may reflect the differences in complement regulation between the mouse and the human. Alternatively, it may mimic the human situation in which individuals are predisposed to aHUS by a $C F H$ mutation, but require an additional trigger of complement activation (e.g. infection, drugs) for disease to manifest. Further investigation of the heterozygote mouse response to such stimuli will be instructive.

De Jorge et al. [14] have also crossed the $\mathrm{CFH}^{-/-} \Delta 16-$ 20 with a C5-deficient mouse to investigate the role of $\mathrm{C} 5$ activation in the pathogenesis of aHUS. These $\mathrm{C5}^{-/-}$ $\mathrm{CFH}^{-/-} \Delta 16-20$ mice, did not develop aHUS, suggesting a critical role downstream of $\mathrm{C} 3 \mathrm{~b}$ generation in aHUS and providing a rationale for use of C5 inhibition (e.g. eculizumab) in the treatment of aHUS.

Heterozygous mutations in complement factor I have also been shown to predispose to aHUS. The factor I knockout mouse $\left(\mathrm{CFI}^{-/-}\right)$shows uncontrolled alternative pathway activation; however, they do not develop aHUS, the pathological picture is of mesangial $\mathrm{C} 3$ deposits with nodular expansion.

Although mutations in membrane cofactor protein $(\mathrm{CD} 46 ; \mathrm{MCP})$ are associated with aHUS, the differences between human and murine complement systems preclude an adequate mouse model. While CD46 is highly expressed on most human tissues including glomerular endothelium, in rodents CD46 is limited to the testes. In place of CD46, rodents express the rodent-specific complement regulatory protein, Crry. Crry has decay-accelerating and co-factor activity. Although embryonically lethal, the Crry knockout mouse $\left(\mathrm{Crry}^{-/-}\right)$can be rescued on a C3-deficient mouse $\left(\mathrm{Crry}^{-/-} \mathrm{C}^{-/-}\right)$. When kidneys from $\mathrm{Crry}^{-/-} \mathrm{C}^{-/-}$mice are transplanted into mice with a functioning complement system, the kidneys fail due to uncontrolled complement activation, principally in the tubulointerstitium. Although not the pathological picture of HUS, this confirms the necessity of Crry in the mouse kidney and highlights the differences between human and murine complement systems.

\section{ADAMTS13 Knockout Models of TTP}

Thrombotic thrombocytopenic purpura has recently been shown to be a disorder of von Willebrand Factor (vWF) regulation [3]. In response to vascular injury, stored vWF is released from endothelial cells as ultra large multimers (UL-vWF). However, some UL-vWF remain associated with the endothelial surface, providing 
binding sites for platelets and possibly other blood components such as leukocytes. The presence of UL-vWF may lead to spontaneous platelet aggregation in the circulation or on the endothelial cell surface if it is not rapidly processed by the metalloprotease ADAMTS13. The discovery of ADAMTS13 as crucial to the pathogenesis of TTP led to two groups generating knockout mice in an attempt to produce an animal model of TTP resembling the rare human Upshaw-Schulman syndrome.

Motto et al. [15] produced an ADAMTS13 knockout in two strains of mice. On the initial background (mixed genetic background C57BL/ 6 and $129 \times 1 / \mathrm{Sv}$ ), which was subsequently shown to express a truncated form of ADAMTS 13, there was complete loss of ADAMTS13 activity. vWF-mediated interactions between platelets and vascular endothelium were prolonged compared to wild type. Despite this, there was no evidence of a thrombotic microangiopathy and no difference in survival compared to wild type. When this ADAMTS $13^{-/-}$mouse was crossed onto the genetically distant CASA/Rk mice (which express full length ADAMTS13), a number of the mice developed spontaneous thrombocytopenia with increased mortality. These mice had the vWF-rich and fibrin-poor hyaline thrombi characteristic of TTP in the small vessels of multiple organs, including the kidney. When these susceptible CASA/Rk ADAMTS13 ${ }^{-/}$mice were exposed to IV Stx, most developed a picture consistent with TTP that was not seen in the CASA/Rk ADAMTS13 $^{+/+}$mice.

As the CASA/Rk mice have higher plasma vWF levels than the mixed genetic background mouse, it had been proposed that this accounted for the difference in phenotype between these two models. However, early generations of the cross between these two mice produced a random inheritance of vWF-regulatory factors leading to a heterogeneous population of knockouts with a wide range of vWF levels. In these mice, no correlation was seen between plasma vWF and the degree of Stx-induced thrombocytopenia or mortality. This suggests that the non-susceptible strain may contain other genetic differences that may protect against TMA.

Banno et al. [16] subsequently produced an ADAMTS13 knockout on pure strain 129/Sv mice, but these mice failed to show any evidence of thrombotic microangiopathy. The ADAMTS13 ${ }^{-/}$mice did, however, show a UL-VWF multimer pattern, similar to that seen in TTP, compared to the ADAMTS13 $3^{+/+}$which demonstrated a normal multimer pattern. When these ADAMTS13 ${ }^{-/-}$ mice were challenged with the platelet and endothelial agonists, collagen and epinephrine, they developed a se- vere thrombocytopenia compared to controls. However, mortality between the two groups was no different.

Thus, these mice suggest that, in addition to $\mathrm{AD}$ AMTS13 deficiency, additional environmental and genetic triggers are required for TTP to manifest.

In addition to the ADAMTS13 knockout mice models of familial TTP, the ADAMTS $13^{+/+}$(on the non-TTP susceptible mouse strain) was injected with polyclonal rabbit antihuman ADAMTS13 antiserum in an effort to replicate acquired TTP. These mice demonstrated significantly prolonged vWF-mediated platelet-endothelial interactions, similar to the untreated ADAMTS13 ${ }^{-/-}$mice not receiving antiserum [17], but did not develop TTP.

\section{VEGF Models of TMA}

Vascular endothelial growth factor (VEGF) is one of the most important endothelial cell growth factors. In addition to its role in induction and maintenance of normal vascular endothelium it is markedly upregulated in many human tumours. Bevacizumab is a humanised monoclonal antibody against VEGF which, as part of chemotherapy regimens, improves survival rates by preventing angiogenesis. A rare complication of this therapy has been the development of renal TMA. In the kidney, VEGF is constitutively expressed in the glomerular podocytes. To examine the role of VEGF in TMAs, various mouse models of VEGF-A deficiency have been created.

VEGF-A ${ }^{-/-}$and VEGF-A ${ }^{-/+}$mice are embryonic lethal due to major vascular defects. To examine the role of VEGF in the kidney, a podocyte-specific deletion of VEGF was generated in mice. Podocyte-specific homozygotes died perinatally with kidney failure and grossly abnormal glomeruli which lacked mature endothelial cells. Mice with podocyte-specific heterozygosity for VEGF developed endotheliosis and bloodless glomeruli, the renal lesion seen in pre-eclampsia, which progressed to nephrotic syndrome and then renal failure. In contrast, overexpression of the 164 isoform of VEGF-A in podocytes led to a collapsing glomerulopathy, which is the renal lesion seen in HIV-associated nephropathy [18]. Thus, VEGF is critical for the normal development of the glomerulus.

To test the role of VEGF inhibition in mature kidney, Eremina et al. [19] used conditional gene targeting to delete VEGF from the renal podocytes in adult mice. These mice developed typical features of thrombotic microangiopathy with intracapillary thrombi on electron microscopy mirroring the thrombotic microangiopathy seen in patients treated with bevacizumab. This suggests a protective role of VEGF in maintaining glomerular vascular 
integrity and preventing the endothelial cell damage which may result in TMA.

\section{Renal Thrombotic Microangiopathy in a Genetic Hypertension Mouse Model}

Malignant hypertension in humans can result in TMA. Transgenic mice that are hypertensive because of overexpression of the human renin $\left(\mathrm{R}^{+}\right.$mice) and angiotensin $\left(\mathrm{A}^{+}\right.$mice) genes develop renal thrombotic microangiopathy when placed on a high salt diet and/or nitric oxide synthase inhibitor, L-NAME, is added to drinking water. This model may lead to renal TMA by (a) hypertensive endothelial cell injury, (b) inhibition of nitric oxide synthase or (c) direct effect of high salt on renal blood vessels.

\section{What Are the Dangers of Extrapolation from Mice} Models of TMA to Humans?

The simple transfer of knowledge from mice to humans is highly illusive. Because there are so many similarities, there is a tendency to ignore differences and make the assumption that what is true in mice is true in humans. The genomes of mice and humans diverged approximately 65-75 million years ago. Mice and humans differ significantly in terms of size, lifespan and immunological systems [20]. For example, of potential significance to the pathophysiology of TMAs are differences in the balance of leukocyte subsets, chemokine and cytokine receptor expression, inducible nitric oxide synthase and complement regulatory proteins. Additionally, mice are housed in pathogen-free areas, and do not usually have other comorbid conditions. Mice are frequently inbred and the phenotype obtained can be dependent on the strain used, as in the ADAMTS13 $3^{-/}$mouse models. One concern regarding the use of knockout mice is that the development of the immune system, in the absence of a specific protein, may result in the generation of confounding and unanticipated compensatory mechanisms. The use of transgenic mice may to some extent address these issues. This seems to be supported by the evidence from the podocyte conditional knockout of VEGF and the $\mathrm{CFH}^{-/} \Delta 16-20$ mouse, which closely replicated the human conditions they attempted to model. Finally, despite the hope that animal models will allow rapid identification of treatments for TMAs, there are many examples in the literature where therapeutic strategies developed in mouse models have not translated into successful human trials, e.g. blockade of TNF $\alpha$ for treatment of sepsis.

\section{Conclusions}

Using the wide range of genetic and environmental triggers of TMAs a large number of animal models have been developed. The initial small animal models of StxHUS were a poor imitation of the human disease while the large animal models are impractical for most laboratories to use. However, the recent introduction of genetically modified mice have probed specific pathways known to be perturbed in humans and this has provided better models of human disease. These mice may serve as a useful platform for testing novel therapeutic strategies in these important conditions.

\section{Acknowledgements}

A.R. is funded by a Wellcome Intermediate Fellowship, D.K. is a Kidney Research UK training fellow. We apologize for the large quantity of original papers we have been unable to quote due to restrictions in space.

\section{References}

1 Nangaku M, Nishi H, Fujita T: Pathogenesis and prognosis of thrombotic microangiopathy. Clin Exp Nephrol 2007;11:107-114.

-2 Kavanagh D, Richards A, Atkinson J: Complement regulatory genes and hemolytic uremic syndromes. Annu Rev Med 2008;59: 293-309.

-3 Tsai HM: Current concepts in thrombotic thrombocytopenic purpura. Annu Rev Med 2006;57:419-436.
Siegler RL, Obrig TG, Pysher TJ, Tesh VL, Denkers ND, Taylor FB: Response to Shiga toxin 1 and 2 in a baboon model of hemolytic uremic syndrome. Pediatr Nephrol 2003;18:92-96.

Keepers TR, Psotka MA, Gross LK, Obrig TG: A murine model of Hus: Shiga toxin with lipopolysaccharide mimics the renal damage and physiologic response of human disease. J Am Soc Nephrol 2006;17:34043414 .
Paixao-Cavalcante D, Botto M, Cook HT, Pickering MC: Shiga toxin-2 results in renal tubular injury but not thrombotic microangiopathy in heterozygous factor $\mathrm{H}$-deficient mice. Clin Exp Immunol 2009;155:339-347.

Nangaku M, Alpers CE, Pippin J, Shankland SJ, Adler S, Kurokawa K, Couser WG, Johnson RJ: A new model of renal microvascular endothelial injury. Kidney Int 1997;52:182194. 
-8 Ren G, Hack BK, Minto AW, Cunningham PN, Alexander JJ, Haas M, Quigg RJ: A complement-dependent model of thrombotic thrombocytopenic purpura induced by antibodies reactive with endothelial cells. Clin Immunol 2002;103:43-53.

-9 Hashimoto K, Tabata N, Fujisawa R, Matsumura H, Miyazawa M: Induction of microthrombotic thrombocytopenia in normal mice by transferring a platelet-reactive, monoclonal anti-gp70 autoantibody established from MRL/lpr mice: an autoimmune model of thrombotic thrombocytopenic purpura. Clin Exp Immunol 2000;119:4756.

-10 Pickering MC, Cook HT, Warren J, Bygrave AE, Moss J, Walport MJ, Botto M: Uncontrolled C3 activation causes membranoproliferative glomerulonephritis in mice deficient in complement factor $\mathrm{H}$. Nat Genet 2002;31:424-428.

-11 Pickering MC, de Jorge EG, Martinez-Barricarte R, Recalde S, Garcia-Layana A, Rose KL, Moss J, Walport MJ, Cook HT, de Cordoba SR, Botto M: Spontaneous hemolytic uremic syndrome triggered by complement factor $\mathrm{H}$ lacking surface recognition domains. J Exp Med 2007;204:1249-1256.
12 Ferreira VP, Herbert AP, Cortes C, McKee KA, Blaum BS, Esswein ST, Uhrin D, Barlow PN, Pangburn MK, Kavanagh D: The binding of factor $\mathrm{H}$ to a complex of physiological polyanions and $\mathrm{C} 3 \mathrm{~b}$ on cells is impaired in atypical hemolytic uremic syndrome. J Immunol 2009;182:7009-7018.

13 Pickering MC, Cook HT: Translational minireview series on complement factor $\mathrm{H}$ : renal diseases associated with complement factor $\mathrm{H}$ : novel insights from humans and animals. Clin Exp Immunol 2008;151:210-230.

14 de Jorge EG, Paixao-Cavalcante D, Rose KL, Cook HT, Botto M, Pickering MC: C5 activation is required for the development of atypical haemolytic uraemic syndrome in CFH-/-FH delta 16-20 mice. 22nd International Complement Workshop, Basel. Mol Immunol 2008;45:4100.

15 Motto DG, Chauhan AK, Zhu G, Homeister J, Lamb CB, Desch KC, Zhang W, Tsai HM, Wagner DD, Ginsburg D: Shigatoxin triggers thrombotic thrombocytopenic purpura in genetically susceptible ADAMTS13-deficient mice. J Clin Invest 2005;115:27522761.
16 Banno F, Kokame K, Okuda T, Honda S, Miyata S, Kato H, Tomiyama Y, Miyata T: Complete deficiency in ADAMTS13 is prothrombotic, but it alone is not sufficient to cause thrombotic thrombocytopenic purpura. Blood 2006;107:3161-3166.

- 17 Chauhan AK, Motto DG, Lamb CB, Bergmeier W, Dockal M, Plaimauer B, Scheiflinger F, Ginsburg D, Wagner DD: Systemic antithrombotic effects of ADAMTS13. J Exp Med 2006;203:767-776.

18 Eremina V, Sood M, Haigh J, Nagy A, Lajoie G, Ferrara N, Gerber HP, Kikkawa Y, Miner JH, Quaggin SE: Glomerular-specific alterations of VEGF-A expression lead to distinct congenital and acquired renal diseases. J Clin Invest 2003;111:707-716.

-19 Eremina V, Jefferson JA, Kowalewska J, Hochster H, Haas M, Weisstuch J, Richardson C, Kopp JB, Kabir MG, Backx PH, Gerber HP, Ferrara N, Barisoni L, Alpers CE, Quaggin SE: VEGF inhibition and renal thrombotic microangiopathy. N Engl J Med 2008;358:1129-1136.

20 Mestas J, Hughes CC: Of mice and not men: differences between mouse and human immunology. J Immunol 2004;172:2731-2738. 